\title{
Análise Neurofuncional de Recém-Nascidos de Mães Cronicamente Infectadas pelo Toxoplasma Gondii
}

\author{
Neurofunctional Analysis of Newborns of Chronically Infected Mothers by \\ Toxoplasma Gondii
}

\author{
Thaís Bandeira Riesco ${ }^{1}$ \\ Mariza Martins Avelino ${ }^{2}$
}

\section{RESUMO}

Objetivo: Avaliar o desenvolvimento neuropsicomotor de recémnascidos de mães cronicamente infectadas pelo Toxoplasma gondii. Metodologia: A população do estudo foi composta por 79 recém-nascidos. Foi realizada uma entrevista sociodemográfica com as mães por intermédio de um questionário, assim como um exame dos reflexos primitivos nos recém-nascidos. As análises foram realizadas com o auxílio do pacote estatístico SPSS $^{\circledR}$, versão 23. Resultados: A correlação de Spearman mostrou que não existiu uma idade em que as mães tiveram um maior contato com a toxoplasmose e a maioria realizou entre cinco a oito consultas pré-natais. No teste de MannWhitney foi observado que os diferentes métodos usados para avaliar a idade gestacional mostraram valores aproximados, ao comparar os recém-nascidos de mães com toxoplasmose crônica e mães suscetíveis ao protozoário. A ausência do reflexo de fuga ao sufocamento foi encontrada em duas crianças, filhas de mães expostas ao protozoário, porém, pelo teste do Qui-quadrado não foi verificada nenhuma associação entre ausências de reflexos primitivos nos recém-nascidos e a presença do Toxoplasma gondii nas mães. O teste de MannWhitney também foi utilizado na comparação entre o APGAR do primeiro e do quinto minutos dos recém-nascidos, no qual não foi constatada diferença significativa. Conclusão: Os recémnascidos de mães cronicamente infectadas pelo Toxoplasma gondii não apresentaram déficit ou atraso neuropsicomotor.

\section{DESCRITORES}

Toxoplasmose. Toxoplasma gondii. Anticorpos e RecémNascido.

\begin{abstract}
Objective: To assess if there are perceptible changes in the neuropsychomotor examination of newborn children of chronically infected mothers by Toxoplasma gondii. Methodology: 79 newborn children composed the population of the study. A sociodemographic interview with the mothers was carried out through a questionnaire, as well as an examination of primitive reflexes in newborns. The analyses were carried out with the aid of the statistical package SPSS ${ }^{\circledR} 23$. Results: Spearman's correlation showed that there was no age at which mothers had greater contact with toxoplasmosis and most had between five to eight prenatal consultations. In the MannWhitney test, it was observed that the different methods used to assess gestational age showed approximate values when comparing newborns of mothers with chronic toxoplasmosis and mothers susceptible to protozoa. The absence of the suffocation escape reflex was found in two children, daughters of mothers exposed to the protozoan, however, by the Chi-square test, no association was found between the absence of primitive reflexes in newborns and the presence of Toxoplasma gondii in the mothers. The Mann-Whitney test was also used to compare the APGAR of the first and fifth minutes of the newborns, in which no significant difference was found. Conclusions: Newborns of mothers chronically infected with Toxoplasma gondii did not present neuropsychomotor deficit or delay.
\end{abstract}

\section{DESCRIPTORS}

Toxoplasmosis. Toxoplasma gondii. Antibodies and Newborn.

${ }^{1}$ Doutoranda do Programa de Pós-Graduação em Ciências da Saúde da Universidade Federal de Goiás (PPGCS/UFG). Fisioterapeuta. Docente do Curso de Fisioterapia da Universidade Paulista (UNIP) e da Universidade Estadual de Goiás (UEG).

${ }^{2}$ Doutora, Pediatra/Infectologista, Professora Associada da Faculdade de Medicina da Universidade Federal de Goiás (UFG). Programa de Pós-Graduação em Ciências da Saúde (PPGCS), Goiânia, Goiás, Brasil. 
$\mathrm{O}$ desenvolvimento infantil é um processo que tem início na vida intrauterina e engloba o crescimento físico, a maturação neurológica, o comportamento e as esferas cognitivas, social e afetiva da criança ${ }^{1}$.

O sistema motor da criança está em constante evolução e modificação de acordo com a idade cronológica, na qual o ser humano tem uma progressão de suas habilidades motoras que vão se tornando mais específicas e complexas, com uma velocidade maior no primeiro ano de vida ${ }^{1,2}$.

É sabido que o feto, quando em contato com agentes infecciosos, pode apresentar inúmeros problemas e um deles é a restrição do crescimento intrauterino, acarretando uma maior mortalidade por serem os recém-nascidos (RNs) pequenos para a idade gestacional ${ }^{3}$.

Toxoplasma gondii é um protozoário intracelular com alta prevalência entre animais e humanos, nos quais os gatos são seus hospedeiros definitivos e os seres humanos e outros animais hospedeiros intermediários. Está intimamente ligado aos hábitos de vida da população, com uma predominância que varia de 20 a 95\%, a depender ainda das condições climáticas de cada região, considerando que países tropicais têm índices mais elevados da doença. As estimativas apontam para o fato de que um terço da população mundial esteja infectada e que essa seja a segunda infecção intrauterina fetal mais comum ${ }^{4-10}$.

Dos recém-nascidos que têm contato com o protozoário Toxoplasma gondii intraútero, $85 \%$ são assintomáticos, porém alguns podem vir a apresentar até os dois anos de idade lesões oculares variando de $20-45 \%$ e lesões no sistema nervoso central.
Além desses sintomas, $13 \%$ ainda podem desenvolver hidrocefalia e microcefalia, surdez em $26 \%$, cefaléia em $17 \%$ e em $13 \%$, risco de atraso mental ${ }^{11,12}$.

No que tange a infecção crônica da gestante, não existem relatos exatos sobre contaminação fetal, porém sabe-se que mesmo na forma latente os parasitas presentes em cistos teciduais, encontrados principalmente nos músculos esqueléticos, no músculo cardíaco e no sistema nervoso central dos hospedeiros, podem sobreviver por toda a vida e serem reativados em algum momento em casos de pessoas imunocompetentes ${ }^{10,13}$.

Em uma pesquisa desenvolvida em Teerã no Irã, foi comprovado que gestantes infectadas pelo Toxoplasma gondii, teriam uma maior suscetibilidade a doenças psiquiátricas, razão pela qual importa mencionar que o próprio estado gestacional, por ser um período relacionado a muitas mudanças orgânicas, também pode levar a uma depressão do sistema imunológico com possibilidade de reativação da doença ${ }^{14}$.

Nesse contexto, o estudo teve o objetivo de avaliar os filhos de mães cronicamente infectadas pelo Toxoplasma gondii, com a finalidade de identificar se os recém-nascidos poderiam apresentar algum atraso no desenvolvimento neuropsicomotor (DNPM).

\section{METODOLOGIA}

O estudo foi de corte transversal prospectivo, na Cidade de Goiânia, Estado de Goiás, no período de junho de 2015 a junho de 2016.

A população do estudo foi constituída 
de 79 crianças nascidas na Maternidade do Hospital das Clínicas (HC) da Universidade Federal de Goiás (UFG), das quais 41 eram filhas de mulheres cronicamente infectadas pelo Toxoplasma gondii e 38, de mulheres suscetíveis ao protozoário, existindo a aceitação expressa de todas na participação da pesquisa, com a assinatura do Termo de Consentimento Livre e Esclarecido.

A coleta de dados teve início após a aprovação pelo Comitê de Ética em Pesquisa Envolvendo Seres Humanos e Animais do Hospital das Clínicas da Universidade Federal de Goiás (Protocolo n. 1.083.346), de acordo com a Resolução n 466/12 do Conselho Nacional de Saúde.

A escolha dos pacientes foi realizada por conveniência, por meio dos prontuários das gestantes que já se encontravam na maternidade internadas em acompanhamento, seja em trabalho de parto, seja após este. As mães responderam a um questionário sociodemográfico e seus filhos foram examinados após 24 horas de nascidos. Para isso, esses recém-nascidos deveriam estar estáveis e no leito juntamente com a mãe.

A confirmação sorológica dos anticorpos das classes IgM e lgG da mãe foi realizada pelo Laboratório de Análises Clínicas do Hospital das Clínicas, por meio do teste de Imunoensaio de Micropartículas por Quimioluminescência (CMIA), Architect, com referencial de: não reagente $\leq 0,499$, zona cinza 0,500 a 0,599 e reagente $\geq 0,600$.

Foram incluídos na pesquisa os filhos de mulheres que realizaram o prénatal pelo Sistema Único de Saúde, os quais tiveram seus partos no HC/UFG e não possuíam infecções agudas (toxoplasmose, citomegalovírus, sífilis, chagas, vírus da imunodeficiência humana, vírus linfotrópico de células $\mathrm{T}$ humanas, vírus do herpes simples humano, rubéola, hepatites $\mathrm{B}$ e $\mathrm{C}$ ) detectadas pelo exame de pré-natal ou pela triagem do pré-parto. Os recém-nascidos instáveis e que as suas mães possuíam alguma infecção aguda foram excluídos da pesquisa.

As análises foram registradas em um banco de dados do Microsoft Excel e realizadas com o auxílio do pacote estatístico SPSS $^{\circledR}$, versão 23. O nível de significância utilizado foi de $5 \%(p<0,05)$. As variáveis quantitativas foram apresentadas com valores da mediana, média, desvio padrão, máximo e mínimo; e as variáveis qualitativas foram apresentadas em números absolutos e porcentagens.

$\mathrm{Na}$ análise da distribuição das variáveis quantitativas foi usado o teste de normalidade Shapiro-Wilk. Em seguida, foram aplicados testes estatísticos não paramétricos. Na comparação dos valores das idades gestacionais: IG-DUM, IG-USG, Capurro somático e Capurro somático e neurológico entre os grupos (anticorpos antiToxoplasma gondii) e das variáveis APGAR 1 e APGAR 5, foi aplicado o teste de MannWhitney. A análise comparativa dos reflexos dos RNs foi realizada com base no teste do Qui-quadrado e a comparação dos valores IGDUM, IG-USG, Capurro somático e Capurro somático e neurológico com os reflexos, feita por meio do teste de Mann-Whitney.

\section{RESULTADOS}

Um total de 79 recém-nascidos foi examinado após entrevista com as mães 
(avaliados após 24 horas completas do nascimento).

Dos recém-nascidos avaliados, 45 (57\%) eram do sexo feminino e 34 (43\%) eram do sexo masculino. A média de idade das gestantes foi de $27,48 \pm 6,58$. Em relação ao número de consultas pré-natais, verificouse uma média de 7,99 consultas, com um mínimo de 2 e um máximo de 15 consultas. Na variável tipo de gravidez, $97,5 \%$ das mães tiveram gravidez única e, do total de mulheres avaliadas, $54(68,4 \%)$ tiveram parto cirúrgico e $25(31,6 \%)$ parto normal, atendidas em uma Maternidade terciária para gestantes de risco.

Em relação à sorologia para toxoplasmose, realizada após as entrevistas das mães, como forma de confirmação dos resultados no pré-natal, realizada por meio do teste de imunoensaio quimioluminescente (CMIA) no Laboratório de Análises Clínicas do HC/UFG, 51,9\% (41) das puérperas tinham infecção crônica lgG (+) lgM (-), e 48,1\% (38) eram soronegativas $\lg G(-) \lg M(-)$.

$\mathrm{Na}$ Figura 1 foi realizada uma representação da correlação de Spearman entre a idade das mães e o número de consultas pré-natais realizadas pelas mesmas, separado por grupos, de acordo com os exames de anticorpos anti-Toxoplasma gondii. Nessa correlação observou-se que o valor de p não foi significativo, ou seja, não existiu uma idade em que as mães tiveram mais contato com a toxoplasmose e, ao considerar a frequência das consultas pré-natais, a maioria ocorreu no intervalo de 5 a 8 consultas.

Observa-se na Tabela 1 que, ao

Figura1. Comparação entre os grupos das 41 mães com Toxoplasma gondii e das 38 suscetíveis em relação à idade e ao número de consultas pré-natais, na Maternidade do Hospital das Clínicas da Universidade Federal de Goiás, em Goiânia (GO), Brasil

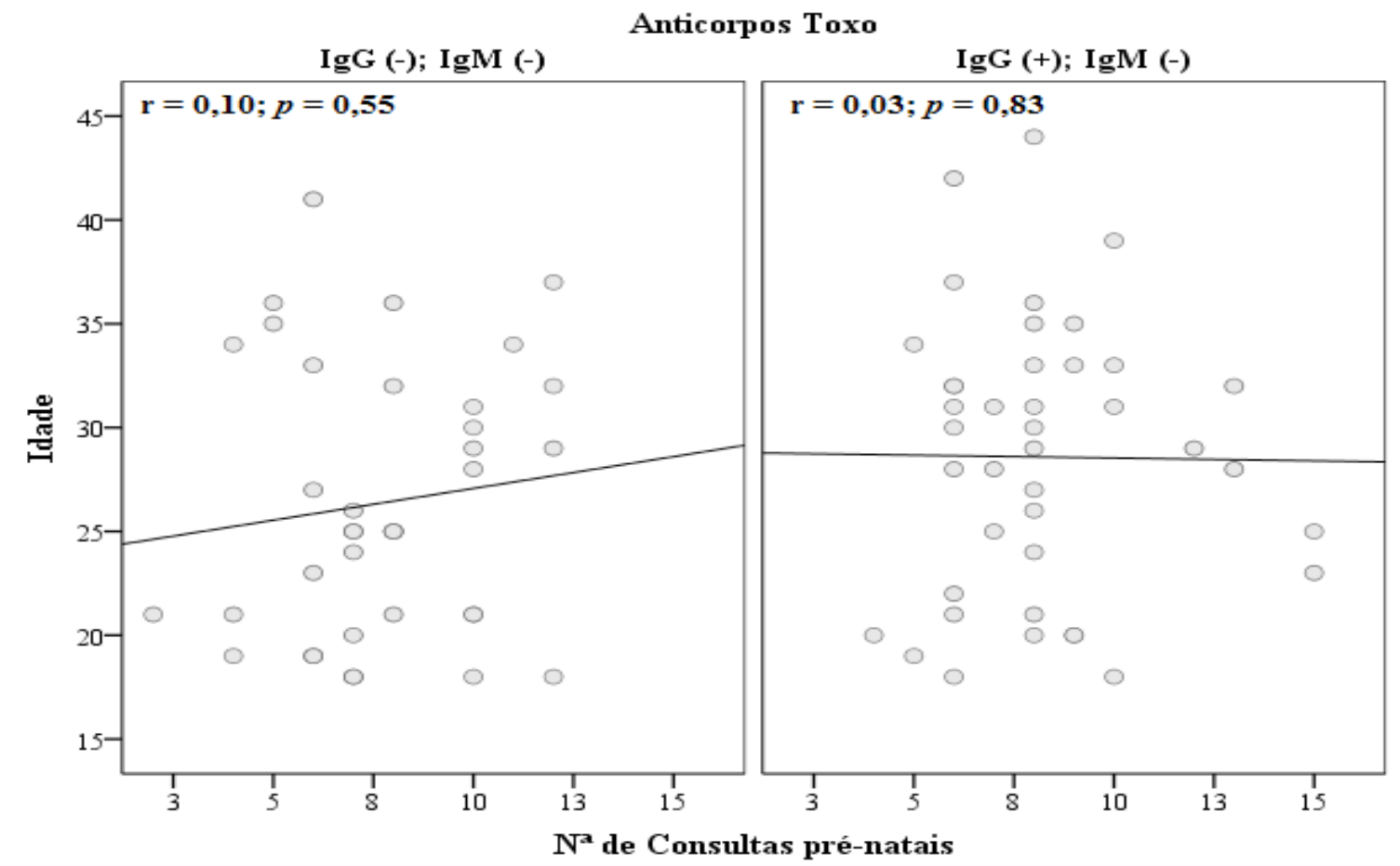

Legenda: Correlação de Spearman; (-): negativo; (+): positivo. 
Tabela 1. Distribuição da Idade Gestacional das 79 puérperas e dos 79 recém-nascidos, por diferentes métodos de avaliação e sua relação com o Toxoplasma gondii na Maternidade do Hospital das Clínicas da Universidade Federal de Goiás, em Goiânia (GO), Brasil

\begin{tabular}{|c|c|c|c|c|}
\hline \multirow{2}{*}{ Variáveis } & \multicolumn{2}{|c|}{ Anticorpos Toxo (Média \pm DP) } & \multirow{2}{*}{ z } & \multirow{2}{*}{$p^{*}$} \\
\hline & $\lg G(-) ; \lg M(-)$ & $\lg G(+) ; \lg M(-)$ & & \\
\hline IG-DUM & $38.17 \pm 1.96$ & $38.50 \pm 2.02$ & 0,79 & 0,42 \\
\hline IG-USG & $38.41 \pm 1.32$ & $38.49 \pm 1.40$ & 0,60 & 0,54 \\
\hline Capurro Somático & $38.48 \pm 1.50$ & $38.77 \pm 1.70$ & 0,80 & 0,42 \\
\hline Capurro Somático Neurológico & $39.35 \pm 1.28$ & $39.57 \pm 1.60$ & 0,69 & 0,48 \\
\hline
\end{tabular}

Legenda: Teste Mann-Whitney; (-): negativo; (+): positivo; IG-DUM: Idade Gestacional pela data da última menstruação; IG-USG: Idade Gestacional pela Ultrassonografia.

Tabela 2. Avaliação do exame neurológico de 38 recém-nascidos de mães infectadas com Toxoplasma gondii e 41 de mães suscetíveis, em Goiânia (GO), Brasil

\begin{tabular}{|c|c|c|c|c|}
\hline & & $(\%)$ & & \\
\hline & $\lg G(-) ; \operatorname{Ig} M(-)(38 n)$ & $\operatorname{lgG}(+) ; \lg M(-)(41 n)$ & $X^{2}$ & $p^{*}$ \\
\hline RFS & & & & \\
\hline Ausente & - & $02(4,9)$ & & \\
\hline Presente & $38(100,0)$ & $39(95,1)$ & 1,90 & 0,16 \\
\hline RS & & & & \\
\hline Ausente & $03(7,9)$ & $02(4,9)$ & & \\
\hline Presente & $35(92,1)$ & $39(95,1)$ & 0,30 & 0,58 \\
\hline MR & & & & \\
\hline Ausente & $12(31,6)$ & $11(26,8)$ & 021 & 064 \\
\hline Presente & $26(68,4)$ & $30(73,2)$ & 0,21 & 0,64 \\
\hline RG & & & & \\
\hline Ausente & $06(15,8)$ & $05(12,2)$ & 021 & 064 \\
\hline Presente & $32(84,2)$ & $36(87,8)$ & 0,21 & 0,64 \\
\hline $\mathrm{RPC}$ & & & & \\
\hline Ausente & $02(5,3)$ & $03(7,3)$ & 011 & 070 \\
\hline Presente & $36(94,7)$ & $38(92,7)$ & 0,14 & 0,10 \\
\hline $\mathrm{RM}$ & & & & \\
\hline Ausente & $01(2,6)$ & $01(2,4)$ & & \\
\hline Presente & $37(97,4)$ & $40(97,6)$ & 0,03 & 0,95 \\
\hline RTCA & & & & \\
\hline Ausente & $05(13,2)$ & $03(7,3)$ & & \\
\hline Presente & $33(86,8)$ & $38(92,7)$ & 0,73 & 0,39 \\
\hline RRC & & & & \\
\hline Ausente & $17(44,7)$ & $20(48,8)$ & & \\
\hline Presente & $21(55,3)$ & $21(51,2)$ & 0,13 & 0,71 \\
\hline
\end{tabular}

Legenda: Teste Qui-quadrado; (-): negativo; (+): positivo; RFS: reação de fuga ao sufocamento; RS: reflexo de sucção; MR: marcha reflexa; RG: reflexo de galant; RPC: reflexo dos pontos cardeais; RM: reflexo de moro; RTCA: reflexo tônico cervical assimétrico e RRC: reação de retificação da cervical 
Tabela 3. Avaliação do boletim de APGAR em recém-nascidos de mulheres expostas e não expostas ao Toxoplasma gondii em Goiânia (GO), Brasil

\begin{tabular}{ccccc}
\hline \multirow{2}{*}{ Variáveis } & \multicolumn{2}{c}{ Anticorpos Toxo } & Z & \multirow{2}{*}{$p^{*}$} \\
& $\operatorname{lgG}(-) ; \operatorname{lgM}(-)$ & $\operatorname{lgG}(+) ; \operatorname{lgM}(-)$ & & 1,21 \\
APGAR 1 & $8,79 \pm 0,84$ & $8,66 \pm 0,48$ & 0,22 \\
APGAR 5 & $9,39 \pm 0,54$ & $9,41 \pm 0,50$ & 0,02 & 0,98 \\
\hline
\end{tabular}

Legenda: Teste Mann-Whitney; (-): negativo; (+): positivo; APGAR: Activity, Pulse, Grimace, Appearance e Respiration.

relacionar a IG-DUM e a IG-USG das 79 gestantes e os capurros dos 79 recémnascidos, obtendo-se diferentes métodos de idades gestacionais, não existiu diferença entre as idades gestacionais dos recém-nascidos de mães com Toxoplasma gondii e os filhos das puérperas suscetíveis ao protozoário, com a obtenção de uma IG aproximadamente igual nos diferentes métodos estudados.

$\mathrm{Na}$ Tabela 2, quando comparados os reflexos primários dos recém-nascidos com 24 horas de vida (reação de fuga ao sufocamento - RFS; reflexo de sucção - RS; marcha reflexa - MR; reflexo de Galant - RG; reflexo dos pontos cardeais - RPC; reflexo de moro - RM; reflexo tônico cervical assimétrico - RTCA; e reação de retificação da cervical - RRC) com os grupos de anticorpos, não foi verificada associação entre a ausência dos reflexos nos recém-nascidos e presença do Toxoplasma gondii na mãe.

Na Tabela 3 mostra uma comparação entre o APGAR do primeiro minuto e o do quinto minuto realizados no momento do nascimento nos $79 \mathrm{RNs}$ dos dois grupos da pesquisa de mães com toxoplasmose crônica e suscetíveis ao Toxoplasma gondii, no teste realizado não foram encontradas diferenças significativas.

\section{DISCUSSÃO}

Essa pesquisa não encontrou nenhuma relação entre a idade das mães e a infecção pelo Toxoplasma gondii, contrastando com um estudo feito na Etiópia em que a maioria das 360 mulheres grávidas, positivas apenas para anticorpos da classe lgG tinha idades acima de 35 anos. Entretanto, os achados de nosso estudo refletem uma amostra de gestantes com uma média de idade de 27 anos, como foi o caso dos achados no lêmen, no qual a maioria das 420 gestantes da pesquisa tinha idade maior ou igual a 25 anos. Isso comprova que nos dois estudos a idade de exposição foi de mães mais jovens. Porém, diferente dos resultados observados na pesquisa em discussão, acredita-se que, no caso do protozoário em questão, quanto maior a idade em anos, maior o risco de soropositividade, tendo em vista a maior exposição, conforme se verifica na maior parte de países desenvolvidos ${ }^{4,5,10}$.

No caso da Cidade de Goiânia onde a pesquisa foi conduzida verificou-se que a média de idade em que as mulheres apresentaram positividade ao protozoário não difere de outros estudos locais, como foi o caso de um estudo conduzido na mesma localidade, no qual 672 gestantes de uma 
amostra de 1.007 tinham idade menor ou igual a 30 anos $^{15}$.

Em relação às consultas de pré-natais realizadas pelas puérperas, a média foi de 7,99 consultas e, considerando que o Ministério da Saúde preconiza um mínimo de 6 consultas pré-natais, esse achado pode ser considerado satisfatório. Ademais, a maioria dos partos foi realizada por procedimento cirúrgico, que é predominante no Brasil e as gestações tiveram duração de 38 semanas. Esses dados correspondem com a pesquisa realizada em Goiás, por intermédio do levantamento de 87.673 registros de nascimentos, do Sistema de Informação sobre Nascidos Vivos (SINASC) $)^{16,17}$

Observou-se que os diferentes métodos utilizados para a avaliação da idade gestacional, como a data da última menstruação (DUM) e a ultrassonografia gestacional e, nos recém-nascidos, capurro somático e capurro somático neurológico, apresentaram aproximação dos resultados quando comparados, não sendo encontradas diferenças entre as 41 crianças do grupo de mães com Toxoplasma gondii e as 38 do grupo de mães suscetíveis ao protozoário. A DUM quando conhecida e com ciclos regulares é um ótimo indicador de idade gestacional e a ultrassonografia fetal, se realizada até 12 semanas de gestação, fornecerá informações precisas $^{18}$.

Quanto à análise do exame neurológico em relação aos reflexos primitivos, não foram observadas diferenças entre a ausência deles em crianças cujas mães tinham exposição anterior a gestação ao Toxoplasma gondii, em comparação aos filhos de mulheres não expostas ao referido agente infeccioso.
No entanto, a ausência do reflexo de fuga ao sufocamento só foi observada entre os filhos de mães com o protozoário. Esse reflexo está presente em neonatos com o tono da musculatura cervical normal e sua ausência pode indicar possível lesão cerebral neurológica. Esse achado nos leva a deduzir sobre a importância da testagem de avidez para toxoplasmose entre mulheres crônicas, a fim de investigar a possibilidade de infecções agudas em mães com baixa avidez. Essa descoberta poderia proporcionar a verificação da existência de infecção congênita, mesmo que silenciosa e oportunizar o acompanhamento do recémnascido em serviços de referência para doenças infecciosas, com o intuito de dirimir consequências neurológicas advindas da exposição intraútero ao protozoário ${ }^{19}$.

Em relação aos outros reflexos primitivos testados, os recém-nascidos estudados não apresentaram alterações relevantes. Tais reflexos são adquiridos pela criança ainda na vida fetal e deixam de ser predominantes por volta de quatro a seis meses de idade dando lugar a reações posturais as quais serão importantes por toda a vida desse novo ser. A reação de retificação cervical (RRC) não é obrigatória no $\mathrm{RN}$, fato importante a descrever, pois no estudo em questão quase metade dos RNs de ambos os grupos não foram capazes de realizar a RRC. Contudo, os reflexos de fuga ao sufocamento, sucção, pontos cardeais, galant, marcha reflexa, Moro e reflexo tônico cervical assimétrico (RTCA) devem em situações normais estar presentes ao nascimento ${ }^{19-21}$. Em ambos os grupos avaliados as crianças tiveram ausência 
desses reflexos, apesar de não ter sido um número expressivo estatisticamente, seria importante o acompanhamento das mesmas por um profissional da fisioterapia, que poderia, de maneira precoce, descobrir e tratar atrasos neurológicos e motores nesses recém-nascidos, mesmo que de pequena magnitude, objetivando um desenvolvimento neuropsicomotor satisfatório.

$\mathrm{O}$ fato de o $\mathrm{RN}$ estar assintomático ao nascimento não impede o aparecimento de problemas futuros em casos de exposição ao Toxoplasma gondii intraútero. Isso pode acontecer quando as mães são contaminadas na gestação por toxoplasmose aguda ou sofrem uma reativação da infecção toxoplásmica. Uma vez que se sabe que a gravidez pode levar a uma possível reativação da infecção, devido as alterações imunológicas e hormonais comuns da gestação, caso esse protozoário esteja no útero, possivelmente poderia atravessar a placenta e infectar 0 feto $^{22,23}$.

No caso da infecção crônica, não se conhecem possíveis repercussões fetais, que poderiam expressar alterações no exame neurológico do recém-nascido, fato esse observado no estudo em questão. Porém, pesquisas recentes, ao realizarem o teste de avidez de toxoplasmose lgG em gestantes com resultados para IgM negativos, identificaram em alguns exames baixa avidez, que significa infecção recente, o que poderia levar a uma exposição do feto a infecção intraútero. Assim, para diferenciar infecção aguda de crônica esse teste é imprescindível e deveria ser prioridade no pré-natal, a fim de poupar o feto de possíveis sequelas relacionadas à toxoplasmose. Como esse teste não é realizado com frequência, certamente não se pode precisar se de fato os recém-nascidos da pesquisa nasceram apenas assintomáticos à doença toxoplásmica ou realmente não foram expostos ao protozoário intraútero ${ }^{13,24}$.

Evidências apontam para o fato de que a toxoplasmose latente pode ainda estar relacionada a distúrbios neuropsiquiátricos, como no caso da depressão, ansiedade e até esquizofrenia, podendo deixar o sistema imunológico da gestante comprometido e este não conseguir responder eficientemente ao combate de infecções ou mesmo reinfecções ${ }^{14,25}$. No que se refere aos fatores limitantes, há que se mencionar o fato de a pesquisa ter se embasado em um número reduzido de gestantes e recém-nascidos avaliados, tendo em vista que a maternidade na qual o estudo foi conduzido é referência em doenças maternas e possui poucos leitos. Sendo assim, recomenda-se que outros estudos sejam feitos em uma maternidade de grande porte com um maior número de indivíduos e por um tempo mais longo.

Outrossim, também figurou como limitação do estudo o longo período a que as gestantes ficaram submetidas ao trabalho de parto, até que fosse decidido pela realização do parto cirúrgico, o que acarretava à gestante e ao feto um sofrimento desnecessário e evitável, se a maternidade tivesse equipes melhor preparadas para avaliar cada gestante em trabalho de parto individualmente sem tanta demora no serviço, isso poderia reduzir danos irreversíveis, tanto à puérpera, quanto ao recém-nascido. 


\section{CONCLUSÃO}

No presente trabalho não foi possível constatar sinais de eventuais acometimentos neurológicos em recém-nascidos de puérperas com toxoplasmose crônica, quando comparados com os filhos de mães suscetíveis a doença.

Contudo, os achados da pesquisa apontam para a relevância de se acompanhar recém-nascidos com ausência de reflexos primitivos, pois estes deveriam estar presentes em situações de normalidade. Essa assistência tem o intuito de dirimir possíveis comprometimentos no desenvolvimento neuropsicomotor, principalmente durante o primeiro ano de vida dessa criança, marcado por grandes aquisições motoras relacionadas a uma maturação acelerada do sistema nervoso. É nessa fase da vida em que ocorre uma grande possibilidade de aparecimento de dificuldades relacionadas a um possível atraso do DNPM.

Essa pesquisa observou ainda a importância de se realizar o teste de avidez de gestantes com toxoplasmose crônica, IgG positivo, para que em resultados de baixa avidez seja conduzido o tratamento apropriado a essas gestantes com infecção aguda, com o intuito de evitar a infecção congênita ou diminuir seus efeitos no feto.

\section{REFERÊNCIAS}

1. Paixão MCM. Bases do desenvolvimento sensório-motor. In: Lanza FC, Gazzotti MR, Palazzin A. Fisioterapia em pediatria e neonatologia: da uti ao ambulatório. 2.ed. Barueri: Manole. 2019; 5:68-83.

2. Alves CIS, Medeiros JG, Carriço LA, Arraes NM, Alencar RF, Melo AN. Confiabilidade inter-observadores da Escala de Saint-Anne Dargassies na avaliação neurológica de recém-nascidos pré-termo sadios. Rev Bras de Saúde Matern Infant. 2018; 18(1):103-112.

3. Kesavan K, Devaskar SU. Restrição de crescimento intrauterino. Pediatric Clinics of North America. 2019; 66(2):403-423.

4. Teweldemedhin M, Gebremichael A, Geberkirstos G, Hadush H, Gebrewahid T, Asgedom SW, Gidey B, Asres $\mathrm{N}$, Gebreyesus H. Seroprevalence and risk factors of Toxoplasma gondii among pregnant women in Adwa district, northern Ethiopia. BMC Infect Dis. 2019;19(327):1-9.

5. Al-Adhroey AH, Mehrass AAO, Al-Shammakh AA, Ali AD, Akabat MYM, Al-Mekhlafi HM. Prevalence and predictors of Toxoplasma gondii infection in pregnant women from Dhamar, Yemen. BMC Infect Dis. 2019; 19(1089):1-9.

6. Bigna JJ, Tochie JN, Tounouga DN, Bekolo AO, Ymele NS, Youda EL, Sime OS, Nansseu JR Global, regional, and country seroprevalence of Toxoplasma gondii in pregnant women: a systematic review, modelling and meta-analysis, Sci Rep. 2020; 10(12102):1-10.

7. Paquet $\mathrm{C}$, Yudin MH. Toxoplasmose pendant la grossesse: Prévention, dépistage et traitement J Obstet Gynaecol Can. 2018; 40(8):694-702.

8. Bobić B, Villena I, Stillwaggon E. Prevention and mitigation of congenital toxoplasmosis. Economic costs and benefits in diverse settings. Food and Waterborne Parasitol. 2019; 16:1-11.

9. Moura IPS, Ferreira IP, Pontes NA, Bichara CNC. Conhecimento e comportamento preventivo de gestantes sobre Toxoplasmose no município de Imperatriz, Maranhão, Brasil. Cienc \& Saúde Colet. 2019; 24(10):3933-3946.

10. Silveira MB, Carneiro Filho MP, Oliveira SR, Oliveira KR, Nascente FM, Rezende HHA, Castro AM, Avelar JB. Soroprevalência e fatores de risco para toxoplasmose em gestantes na região metropolitana de Goiânia, Goiás, Brasil. Braz. J Hea Rev. 2020; 3(1): 729-746.

11. Fonseca ZC, Rodrigues IMX, Melo NC, Avelar JB, Castro AM, Avelino MM. IgG Avidity Test in Congenital Toxoplasmosis Diagnoses in Newborns. Pathogens. 2017; $6(2): 1-11$.

12. Khan K, Khan W. Congenital toxoplasmosis: An overview of the neurological and ocular manifestations. Parasitol Int. 2018; 67:715-721.

13. Khademi SZ, Ghaffarifar F, Dalimi A, Davoodian P, Abdoli A. Prevalence and Risk Factors of Toxoplasma gondii Infection 
among Pregnant Women in Hormozgan Province, South of Iran. Iran J Parasitol. 2019; 14(1):167-173.

14. Shiadeh MN, Rostami A, Pearce BD, Gholipourmalekabadi M, Newport DJ, Danesh M, Mehravar S, Seyyedtabaei SJ. The correlation between Toxoplasma gondii infection and prenatal depression in pregnant women. Eur J Clin Microbiol Infect Dis. 2016; 35:1829-1835.

15. Rostami A, Riahi SM, Gamble HR, Fakhri Y, Shiadeh MN, Danesh M, Behniafar H, Paktinat S, Foroutan M, Hotez PJ, Gasser, RB. Global prevalence of latent toxoplasmosis in pregnant women: a systematic review and metaanalysis. Clin Microbiol and Infect. 2020; 26(6):673-683.

16. Ávila ALA, Mendonça Júnior JF, Motta LHR, Nascente Júnior REP, Silva TA, Guerra HS. Perfil epidemiológico das puérperas e nascidos vivos no estado de Goiás. Rev Educação em Saúde. 2019; 7(1):90-99.

17. Giordano JC, Nascimento SL, Godoy-Miranda AC, Surita FG. The misleading choice for safer births in Brazilian's most developed region: A cross sectional study. The Journal of Maternal-Fetal \& Neonatal Medicine. 2017; 46(3):509-516.

18. Wilkins-Haug LE, Heffner LJ. Avaliação Fetal e Diagnóstico Pré-Natal. In: Cloherty JP, Eichenwald EC, Hansen AR, Stark AR. Manual de neonatologia. 7.ed. Rio de Janeiro: Guanabara Koogan. 2019; 1:1-8.

19. Paixão MCM. Bases do desenvolvimento sensório-motor. In: Lanza FC, Gazzotti MR, Palazzin A. Fisioterapia em pediatria e neonatologia: da uti ao ambulatório. 2. ed. Barueri: Manole, 2019; 5:68-83.

20. Adamović T, Jurišić-Škevin A, Madić D, Sovilj M, Jeličić L, Maksimović S, Subotić M. Head righting reflex in newborns as the predictive factor of early child development: a longitudinal study. Early Child Dev and Care. 2020; 190:113
21. Walker VP. Newborn Evaluation. In: Gleason C, Juul S. Avery's Diseases of the Newborn. 10th. ed. Washington: Elsevier. 2018; 25:289-331.

22. El Bissati K, Levigne P, Lykins J, Adlaoui EB, Barkat $A$ Berraho A, Laboudi M, El Mansouri B, Ibrahimi A, Rhajaou M, Quinn F, Murugesan M, Seghrouchni F, Gómez-Marín JE, Peyron F, McLeod R. Global initiative for congenital toxoplasmosis: an observational and international comparative clinical analysis. Emerging Microbes \& Infect. 2018; 7(1):1-14

23. Odenirana PO, Omolabib KF, Ademola IO. Risk factors associated with seropositivity for Toxoplasma gondii in population-based studies among immunocompromised patients (pregnant women, HIV patients and children) in West African countries, Cameroon and Gabon: a metaanalysis. Acta Tropica. 2020; 209(105544).

24. Shojaee S, Rahbari AH, Keshavarz $H$. The Relations Between Anti-Toxoplasma IgG and IgM Antibodies With Avidity Index. Crescent Journal of Medical and Biological Sciences. 2017; 4(4):1-3.

25. Bay-Richter C, Buttenschøn HN, Mors O, Eskelund A Budac D, Kærlev L, Wegener G. Latent toxoplasmosis and psychiatric symptoms - A role of tryptophan metabolism? J Psychiatr Res. 2018; 110:45-50.

\section{CORRESPONDÊNCIA}

Thaís Bandeira Riesco

Rua C 86, Quadra 20, Lote 01/04, Vila Alpes, Residencial Mirage. CEP: 74310270.

Goiânia, Goiás, Brasil.

E-mail: thaisriesco@gmail.com. 